\title{
Avaliação clínica, laboratorial e perfil eletroforético na determinação do prognóstico de cães hospitalizados
}

\section{Evaluation of clinical, laboratory and electrophoretic profile to determine of the prognosis of hospitalized dogs}

\author{
Andrei Kelliton Fabretti ${ }^{*}$; Inês Cristina de Batista Fonseca'; \\ Iauani Varison Costa Pancieri³; Fabíola Cardoso Knupp ${ }^{3}$; \\ Danilo Marques Bordini ${ }^{3}$; Patrícia Mendes Pereira ${ }^{1}$
}

\begin{abstract}
Resumo
Várias são as doenças que podem acometer os animais de estimação. Reconhecer os enfermos em risco de óbito permitiria intervenções médicas precoces e o estabelecimento de protocolos de monitoração diferenciados, aumentando a sobrevida. O objetivo deste trabalho foi analisar o potencial para marcadores de prognóstico de variáveis clínicas e laboratoriais acessíveis em 185 cães hospitalizados. Os animais foram alocados em dois grupos: os que obtiveram alta e os que foram a óbito, sendo o estado nutricional (EN) clínico definido pela interpretação do escore de condição corporal (ECC), escore de massa muscular (EMM) e índice de massa corpórea (IMC). Foram avaliadas as seguintes variáveis laboratoriais: eritrograma, contagem total de leucócitos, concentração de fibrinogênio, glicemia, cálcio, fósforo, magnésio, colesterol, albumina, proteína total e perfil proteico sérico definido por eletroforese. Foram indicativos de mau prognóstico: baixos valores de ECC, baixos valores de EMM, número total de linfócitos reduzido, hiperfosfatemia, concentrações reduzidas de albumina e de proteína total e diminuição da fração betaglobulina, na eletroforese. Foram indicadores de bom prognóstico: ECC normal ou elevado, adequado EMM e elevação discreta da fração alfa2-globulina, na eletroforese. Os autores não consideraram a glicemia, colesterol e o cálcio como indicadores de prognóstico.

Palavras-chave: Paciente crítico, bioquímica sérica, sobrevida
\end{abstract}

\begin{abstract}
There are several diseases that can affect pets. Recognize the infirm at risk of death would allow early medical interventions and the establishment of different monitoring protocols, increasing survival. The objective of this work was to analyze the potential for prognostic markers of clinical and laboratory accessible variables in 185 hospitalized dogs. The animals were divided into two groups: those who had hospital outcome and those who have died, being the clinical nutritional status (NS) defined by the interpretation of body condition score (BCS), muscle mass score (MMS) and body mass index (BMI). The following laboratory variables were assessed: blood cell count, total leukocyte count, fibrinogen concentration, glucose, calcium, phosphorus, magnesium, cholesterol, total protein, albumin and serum protein profile defined by electrophoresis. In the present study, were indicative of poor prognosis: low values of BCS, low values of MMS, decreased total number of lymphocytes, hyperphosphatemia,
\end{abstract}

\footnotetext{
${ }^{1}$ Profs. Drs., Dept ${ }^{\circ}$ de Clínicas Veterinárias, Universidade Estadual de Londrina, UEL, Londrina, PR. E-mail: fabretti@uel.br; pmendes@uel.br

2 Prof $^{\mathrm{a}} \mathrm{Dr}^{\mathrm{a}}$, Dept ${ }^{\mathrm{o}}$ de Agronomia, UEL, Londrina, PR. E-mail: inescbf@uel.br

${ }^{3}$ Discentes do Curso de Graduação em Medicina Veterinária, UEL, Londrina, PR. E-mail: iauani89@hotmail.com; fabiknupp@ gmail.com; masanadmb@msn.com

Autor para correspondência 
reduced concentrations of albumin and total protein and betaglobunin fraction, in electrophoresis. Good prognostic indicators were: normal or high BSC, appropriate MMS and discreet lift of alpha2-globulin fraction on electrophoresis. The authors did not consider blood glucose, cholesterol and calcium as prognostic indicators.

Key words: Critical patients, serum biochemistry, survival

\section{Introdução}

Vários são os animais atendidos em clínicas ou hospitais veterinários que podem estar acometidos por doenças sistêmicas que se repercutem em mal estar e podem colocar a vida destes em risco. Estes pacientes sofrem processos infecciosos, sepse, traumas ou resposta inflamatória sistêmica, secundária a agressões variadas; além de alterações metabólicas importantes, prejudiciais a homeostasia natural. A resposta do organismo é o aumento do catabolismo, resultado de uma maior liberação de mediadores endógenos, como hormônios do estresse e citocinas (BRUNETTO, 2006). Com o passar do tempo, o estado catabólico pode desencadear desnutrição, com perda de massa adiposa e muscular, disfunções sistêmicas, queda na resposta imune e comprometimento da capacidade de cicatrização tecidual (VANNUCCHI; UNAMUNO; MARCHINI, 1996; JEEJEEBHOY, 2000; BRUNETTO, 2006; MEIJERS et al., 2010).

Descreve-se, segundo a literatura, que até 50\% dos animais hospitalizados estão subnutridos, o que está associado, direta ou indiretamente, à maior taxa de infecções hospitalares, maior tempo e custo de internamento, maiores taxas de complicações e reinternação, assim como o aumento na mortalidade (CHANDLER; GRECO; FETTMAN, 1992; REILLY et al., 1995; VANNUCCHI; UNAMUNO; MARCHINI, 1996; REMILLARD; ARMSTRONG; DAVENPORT, 2000; LOGAN; HILDEBRANDT, 2003). Dessa forma, o reconhecimento dos animais hospitalizados com mau prognóstico permitiria intervenções precoces e o estabelecimento de protocolos de monitoração diferenciados, aumentando a sobrevida (SPEROTTO; SPINELLI, 2010).
Além disso, os profissionais da saúde estão sendo cada vez mais questionados em relação ao prognóstico dos pacientes e, em geral, existe uma tendência ao pessimismo (COCKCROFT, 2007; RABELO; ARNOLD; ALSUA, 2009). A exatidão do prognóstico é de extrema importância, pois a superestimação do risco de óbito faz com que o médico deixe de utilizar todas as opções diagnósticas e terapêuticas (POSES et al., 1991; RABELO; ARNOLD, 2009).

Foi comprovado que o prognóstico possui maior acurácia quando apoiado em modelos de predição de taxas de alta eóbito(COCKCROFT, 2007; RABELO; ARNOLD, 2009; RABELO; ARNOLD; ALSUA, 2009). Os índices prognósticos são criados a partir de dados coletados de uma determinada população, com seu perfil próprio de enfermidades que, após analisados, são extrapolados para outras realidades. O desempenho de um índice genérico pode divergir quando aplicado em diferentes populações, estados mórbidos e épocas (COCKCROFT, 2007; ALVES et al., 2009; RABELO; ARNOLD, 2009).

A utilização de marcadores de prognósticos auxiliam a selecionar adequadamente os animais que necessitam de cuidados intensivos, permite observar a evolução do paciente e a prever quais terão despesas médicas mais onerosas, permite avaliar o desempenho de um serviço de internamento e embasa as indicações pela eutanásia (COCKCROFT, 2007; RABELO; ARNOLD, 2009; SILVA et al., 2014).

A medicina humana dispõe de vários escores (APACHE - Acute Physiology and Chronic Health Evaluation, TISS - Therapeutic Intervention Scoring System, SAPS - Simplified Acute Physiology Score, MPM - Mortality Probability Model) para predizer 
a mortalidade de pacientes gravemente enfermos (MINNE; ABU-HANNA; DE JONGE, 2008; ALVES et al., 2009; RABELO; ARNOLD, 2009; SILVA et al., 2014). Escores prognósticos similares foram desenvolvidos para aplicação em medicina veterinária (SPI - Survival Prediction Index, ATT - Animal Trauma Triage Scoring, RICO - Rapid Intensive Care Outcome), porém sua aplicação em hospitais ou clínicas ainda é limitada e pouco difundida (KING et al., 2001, RABELO; ARNOLD, 2009; RABELO; ARNOLD; ALSUA, 2009).

Dessa forma, opresente trabalho procurou analisar o potencial de variáveis clínicas e laboratoriais acessíveis como marcadoras de prognóstico em cães hospitalizados. Para a realização desse estudo, os animais tiveram a idade, tempo de internamento, variáveis clínicas, laboratoriais e eletroforéticas associadas com a taxa de alta e óbito.

\section{Material e Métodos}

O experimento foi realizado com os cães internados no setor da Clínica Médica de Animais de Companhia, do Hospital Veterinário da Universidade Estadual de Londrina (HVUEL). Os exames laboratoriais foram realizados no Laboratório de Patologia Clínica, da mesma instituição. A Comissão de Ética no Uso de Animais da UEL aprovou todos os procedimentos desse estudo (CEUA processo $n^{\circ} 31 \backslash 11$ ).

Foram considerados para a inclusão os cães internados no referido setor, no período de agosto de 2011 a agosto de 2012. Para criar informações que aportasse qualquer segmento da população, não houve exclusão baseados em raça, gênero, idade ou motivo de internação. Os pacientes com desidratação clínica acima de 7\% (BATEMAN; CHEW, 2006), os agressivos, dispneicos ou com menos de $1,5 \mathrm{~kg}$ foram excluídos do estudo.

Assim foram avaliados 185 animais, destes, $52,97 \%$ (98/185) eram machos e 47,3\% (87/185) fêmeas. A idade dos cães variou de um mês a 17 anos (mediana de seis anos). O peso variou de dois a $55,4 \mathrm{~kg}$ (mediana de $8,3 \mathrm{~kg}$ ). As raças encontradas foram Poodle (13), Boxer (8), Shitzu (8), Cocker spaniel inglês (7), Tequel (7), Pitbull (5), Labrador retriever (5), Pinscher (5), Dálmata (4), Lhasa apso (4), Rottwailer (3), Pastor belga (2), Maltês (2), Schnauzer (2), e um exemplar das raças: Basset hound, Pastor Alemão, White highland west terrier, Yorkshire, Chow-chow, Golden retriever e Akita, além de 104 cães sem raça definida.

As suspeitas diagnósticas foram agrupadas da seguinte forma: doenças gastroentéricas (70), do sistema urinário (48), infecciosas (42), neurológicas (25), hemolinfáticas (16), respiratórias (10), oncológicas (9), reprodutivas (7), endócrinas (4), dermatológicas (4), insuficiência cardíaca (4), doenças imunomediadas (3), esquelética (3) e oftálmica (1). A grande diversidade de raças e motivos de internação impediu a análise individual do prognóstico para cada afecção ou raça.

Os animais foram distribuídos em dois grupos: os que obtiveram alta da hospitalização (G1) e os que foram a óbito durante esse período (G2). Para graduar o estado nutricional (EN) clínico dos cães foi realizado um exame físico específico. Os animais foram avaliados em relação ao peso, escore de condição corporal (ECC), escore de massa muscular (EMM) e índice de massa corpórea (IMC). Foram também registrados os seguintes dados: raça, idade, gênero, período de internação (em dias) e motivo da internação e/ou doença.

Para a pesagem dos animais foi utilizada a balança eletrônica, modelo LD $200 *$ (Fabricante Lucastec Balança Eletrônica Ltda). Para a determinação do ECC, os cães foram classificados na escala de Laflamme (1997), que consiste em graduações de 1 a 9. A determinação do EMM foi realizada de acordo com a técnica descrita por MICHEL (2009). Para o cálculo do IMC, utilizou-se a fórmula padrão: peso corporal( $\mathrm{kg}) /[\text { estatura }(\mathrm{m})]^{2}$. A mensuração da estatura dos cães e a classificação foram realizadas de acordo com a técnica descrita por Muller, Pinheiro e Mendonça (2008). 
Foram colhidos $5 \mathrm{~mL}$ de sangue da veia jugular, em uma única ocasião, para a realização das análises laboratoriais. $\mathrm{O}$ eritrograma e a contagem total de leucócitos foram efetuados pelo aparelho BC-2800VET, da marca Mindray ${ }^{\circledR}$. A contagem diferencial de leucócitos foi realizada através da microscopia óptica, a partir de um esfregaço sanguíneo, corado pelo panótico rápido para hematologia. As mensurações plasmáticas foram realizadas no aparelho de bioquímica Siemens Dimensions $R X L^{\circledR}$. A concentração de fibrinogênio foi determinada pelo método de precipitação pelo calor (KANEKO; SMITH, 1967).

Para a realização das eletroforeses, o soro foram dispostos no gel de agarose $\left(\right.$ Celmgel $\left.^{\circledR}\right)$ e imersos em solução tampão Tris-Glicina, de pH 9,5, dentro da encubadora CELM ${ }^{\circledR}$ C-90 (série 235). Em seguida, foram submetidos a uma corrente elétrica gerada pelo aparelho CELM $^{\circledR}$ FEA 250 (série 158), por vinte minutos. Após esse período, as placas foram coradas e as concentrações das frações proteicas foram definidas por densitometria, pelo aparelho Canoscan 640P ex, executado por um programa computadorizado. A equipe do laboratório não teve acesso aos resultados do exame físico e grupo experimental dos animais dos quais o sangue era proveniente, estando cegos para a condição nutricional dos cães estudados.

A associação entre o prognóstico dos animais hospitalizados e as variáveis clínicas, laboratoriais e de eletroforese, classificadas em categorias, foi analisada pelo teste Qui-quadrado $\left(\chi^{2}\right)$ ou Teste Exato de Fisher, quando ocorreu frequência esperada (Eij) menor do que 5. No caso de variáveis contínuas, o teste utilizado foi o de Mann-Whitney $(\mathrm{M}-\mathrm{W})$. A opção pelo teste não paramétrico foi devido a não normalidade dos dados, testada pelo teste Shapiro-Wilk. As variáveis categóricas foram apresentadas em frequências absoluta e percentual e as contínuas, em média, desvio-padrão, mediana e quartis 3 e 1 . As análises foram feitas no SAS versão 8.2 (software Institute Inc. Cary, N.C.).

Não foram considerados nesta avaliação a glicemia, colesterol, cálcio e proteína total como indicadores de prognóstico, pois não houve desvio significativo em relação aos valores de referência, fato notado após a análise criteriosa e interpretação dos dados.

\section{Resultados e Discussão}

Os resultados indicaram que dos animais estudados, $126(68,1 \%)$ tiveram alta e $59(31,8 \%)$ foram a óbito. Não foi notada a associação entre o peso, idade, duração da hospitalização e IMC com o desfecho dos animais (Tabelas 1 e 2). Divergindo deste achado, Brunetto (2006) avaliou a associação entre o tempo de internamento e prognóstico de cães e gatos hospitalizados (522 animais) e notou que os pacientes que ficavam mais tempo hospitalizados tinham maior taxa de alta, pois os óbitos ocorriam em poucos dias de hospitalização.

Em humanos, o IMC está associado ao prognóstico, quanto mais elevado for o IMC maior é a mortalidade dos pacientes (MONTEIRO et al., 2000; BEGHETTO, 2007). Por outro lado, valores baixos sugerem desnutrição e maior morbimortalidade (MONDINI; MONTEIRO, 1998). Ou seja, os dois extremos do IMC estão associados com pior prognóstico (VANNUCCHI; UNAMUNO; MARCHINI, 1996; BEGHETTO, 2007). 
Tabela 1. Associação do desfecho com o peso (porte), em frequência (\%), para cães hospitalizados, no período de agosto de 2011 a agosto de 2012, no setor de Clínica Médica de Animais de Companhia, do Hospital Veterinário da Universidade Estadual de Londrina. Londrina, 2013.

\begin{tabular}{|c|c|c|c|}
\hline Variável & $\begin{array}{c}\text { G1 (Alta) } \\
\mathrm{n}=125\end{array}$ & $\begin{array}{c}\text { G2 (Óbito) } \\
\text { n=59 }\end{array}$ & Análise \\
\hline Peso 1-10kg & $67(71,28 \%)$ & $27(28,72 \%)$ & \\
\hline Peso $>10-25 \mathrm{~kg}$ & $46(64,79 \%)$ & $25(35,21 \%)$ & $\begin{array}{c}\chi^{2}=1,00 * \\
\mathrm{GL}=2 \\
\mathrm{p}<0,605\end{array}$ \\
\hline Peso $>25 \mathrm{~kg}$ & $12(63,16 \%)$ & $7(36,84 \%)$ & \\
\hline
\end{tabular}

* $\chi 2=$ Teste de Qui-quadrado, GL $=$ Grau de Liberdade.

Fonte: Elaboração dos autores.

Tabela 2. Associação do desfecho com a idade, tempo de internação e índice de massa corporal (IMC), em média e mediana (Q3-Q1), para cães hospitalizados, no período de agosto de 2011 a agosto de 2012, no setor de Clínica Médica de Animais de Companhia, do Hospital Veterinário da Universidade Estadual de Londrina. Londrina, 2013.

\begin{tabular}{cccc}
\hline Variável & $\begin{array}{c}\text { G1 (Alta) } \\
\mathrm{n}=125\end{array}$ & $\begin{array}{c}\text { G2 (Óbito) } \\
\mathrm{n}=55\end{array}$ & Análise \\
\hline Idade (meses) & & & $\mathrm{M}-\mathrm{W} *$ \\
Média $\pm \mathrm{DP}$ & $71,98 \pm 51,61$ & $66,32 \pm 52,2$ & $\mathrm{p}=0,562$ \\
Mediana & $72,0(120,0-24,0)$ & $48,0(108,0-22,0)$ & \\
Internação (dias) & $6,16 \pm 3,24$ & $5,7 \pm 3,3$ & $\mathrm{M}-\mathrm{W}$ \\
Média $\pm \mathrm{DP}$ & $5,0(7,0-4,0)$ & $5,0(8,0-3,0)$ & $\mathrm{p}=0,238$ \\
Mediana & $14,59 \pm 8,03$ & $12,96 \pm 4,13$ & $\mathrm{M}-\mathrm{W}$ \\
IMC $\left(\mathrm{kg} / \mathrm{m}^{2}\right)^{1}$ & $13,96(15,61-11,00)$ & $12,59(14,50-10,59)$ & $\mathrm{p}=0,112$ \\
Média $\pm \mathrm{DP}$ & & \\
Mediana &
\end{tabular}

${ }^{1}$ Valores de referência: 11,8 e $15 \mathrm{~kg} / \mathrm{m}^{2}$ (MULLER; PINHEIRO; MENDONÇA, 2008). *Teste de Mann-Whitney.

Fonte: Elaboração dos autores.

Para esse trabalho, os cães estudados, tanto os do grupo G1 quanto G2, tinham IMC indicativo de peso ideal (Tabela 2). Uma possível explicação seria o fato de que, nos pacientes críticos, o peso pode se alterar rapidamente devido à depleção hídrica, sobrecarga ou presença de edema e efusões, mascarando a provável perda de massa muscular ou adiposa. Portanto, o IMC desses pacientes é superestimado (CERVI; FRANCESCHINI; PRIORI, 1998; FONTOURA et al., 2006; MAICÁ; SCHWEIGERT, 2008).

A avaliação do ECC permite inferir a quantidade de tecido adiposo que o paciente possui. É utilizado para determinar o EN do paciente e quantificar os desvios em relação ao peso ideal (LAFLAMME, 1997; WORLD SMALL ANIMAL VETERINARY ASSOCIATION, 2011). Dos animais estudados, $46,49 \%(86 / 185)$ foram considerados magros (ECC 1 a 3), 41,62\% (77/185) estavam no peso ideal (ECC 4 e 5) e 11,89\% (22/185) com sobrepeso (ECC 6 a 9). Os animais magros foram considerados clinicamente desnutridos (Tabela 3 ).

O ECC foi associado significativamente às taxas de alta e óbito $(p<0,01)$ (Tabela 3 e 4$)$. A tabela 4 
ilustra a associação do desfecho com o ECC de forma individual, para cada graduação do escore. As maiores taxas de alta foram observadas nos animais com ECC $6(94,12 \%)$ e 7 (100\%), ou seja, com sobrepeso, enquanto os animais muito magros, com ECC 1 e 2 tiveram as maiores taxas de óbito $(55,56 \%$ e $37,84 \%$, respectivamente). Achados semelhantes foram observados na pesquisa de BRUNETTO (2006).

Tabela 3. Associação do desfecho com o escore de condição corporal (ECC), agrupado em: cães magros (1-3), ideal (4 e 5) e sobrepeso (6-9), em frequência (\%), para cães hospitalizados, no período de agosto de 2011 a agosto de 2012, no setor de Clínica Médica de Animais de Companhia, do Hospital Veterinário da Universidade Estadual de Londrina. Londrina, 2013.

\begin{tabular}{ccccc}
\hline ECC & $\mathbf{1 - 3}$ & $\mathbf{4 - 5}$ & $\mathbf{6 - 9}$ & Análise \\
\hline G1 (Alta) $\mathrm{n}=126$ & $55(63,95 \%)$ & $50(64,94 \%)$ & $21(95,45 \%)$ & $\chi 2=8,61 *$ \\
GL 2 (Óbito) $\mathrm{n}=59$ & $31(36,05 \%)$ & $27(35,06 \%)$ & $1(4,55 \%)$ & \begin{tabular}{c} 
GL $=\mathbf{0 , 0 1 3}$ \\
\hline Total
\end{tabular} \\
& $86(46,49 \%)$ & $77(41,62 \%)$ & $22(11,89 \%)$ & $185(100 \%)$ \\
\hline
\end{tabular}

$* \chi 2=$ Teste de Qui-quadrado, GL $=$ Grau de Liberdade.

Fonte: Elaboração dos autores.

Tabela 4. Associação do desfecho com o escore de condição corporal (ECC), em frequência (\%), para cães hospitalizados, no período de agosto de 2011 a agosto de 2012, no setor de Clínica Médica de Animais de Companhia, do Hospital Veterinário da Universidade Estadual de Londrina. Londrina, 2013.

\begin{tabular}{cccccccccc}
\hline ECC & $\mathbf{1}$ & $\mathbf{2}$ & $\mathbf{3}$ & $\mathbf{4}$ & $\mathbf{5}$ & $\mathbf{6}$ & $\mathbf{7}$ & $\mathbf{8}$ & Análise \\
\hline \multirow{2}{*}{ Alta } & 4 & 23 & 28 & 31 & 19 & 16 & 4 & 1 & \\
& $(44,4 \%)$ & $(62,1 \%)$ & $(70,0 \%)$ & $(63,2 \%)$ & $(67,8 \%)$ & $(94 \%)$ & $(100 \%)$ & $(100 \%)$ & $\mathrm{TF}^{*}$ \\
\multirow{2}{*}{ Óbito } & 5 & 14 & 12 & 18 & 19 & 1 & 0 & 0 & $\mathbf{p}<\mathbf{0 , 0 0 1}$ \\
& $(55,5 \%)$ & $(37,8 \%)$ & $(30,0 \%)$ & $(36,7 \%)$ & $(32,1 \%)$ & $(5,8 \%)$ & $(0,0 \%)$ & $(0,0 \%)$ & \\
\hline Total & $9(4,8)$ & $37(19,8)$ & $40(21,5)$ & $49(26,3)$ & $38(20,4)$ & $17(9,1)$ & $4(2,1)$ & $1(0,5)$ & $185(100)$ \\
\hline
\end{tabular}

* Teste de Fisher.

Fonte: Elaboração dos autores.

Sabe-se que a perda muscular é maior em animais doentes e está correlacionada com desnutrição proteica, imunodeficiência, fraqueza muscular, menor capacidade cicatricial e maior mortalidade (VANNUCCHI; UNAMUNO; MARCHINI, 1996; EVANS et al., 2008; WORLD SMALL ANIMAL VETERINARY ASSOCIATION, 2011). Neste trabalho, 2,2\% (4/185) dos cães apresentaram perda muscular severa, 21,08\% (39/185) perda muscular moderada, $27,6 \%$ perda muscular discreta $(51 / 185)$ e $49,2 \%(91 / 185)$ massa muscular normal (Tabela 5). Corroborando com outros pesquisadores, o EMM foi fortemente associado às taxas de alta e óbito $(\mathrm{p}<0,001)$ e pode-se observar que, quanto mais grave foi a perda muscular, maior foi o risco de óbito.

Os animais clinicamente desnutridos (ECC $\leq 3$ e/ou $E M M \leq 2$ ) apresentaram as maiores taxas de óbito, resultados semelhantes foram observados em outras pesquisas (BRUNETTO, 2006, FERREIRA et al., 2006).

Em relação às variáveis laboratoriais, houve associação estatisticamente significativa do desfecho com o número de linfócitos e com concentrações séricas de glicose, cálcio, fósforo, albumina e proteína total, mas não entre o desfecho com volume globular, concentração de hemoglobina, fibrinogênio, colesterol e magnésio. 
Tabela 5. Associação do desfecho com o escore de massa muscular(EMM), em frequência (\%), para cães hospitalizados, no período de agosto de 2011 a agosto de 2012, no setor de Clínica Médica de Animais de Companhia, do Hospital Veterinário da Universidade Estadual de Londrina. Londrina, 2013.

\begin{tabular}{cccccc}
\hline EMM & $\mathbf{0}$ & $\mathbf{1}$ & $\mathbf{2}$ & $\mathbf{3}$ & Análise \\
\hline Alta $(\mathrm{n}=126)$ & $2(50,00 \%)$ & $18(46,15 \%)$ & $33(64,71 \%)$ & $73(80,22 \%)$ & TF \\
Óbito $(\mathrm{n}=59)$ & $2(50,00 \%)$ & $21(53,85 \%)$ & $18(35,29 \%)$ & $18(19,78 \%)$ & $\mathbf{p}<\mathbf{0 , 0 0 1}$ \\
\hline Total & $4(2,2 \%)$ & $39(21,0 \%)$ & $51(27,6 \%)$ & $91(49,2 \%)$ & $185(100,0 \%)$ \\
\hline
\end{tabular}

* Teste de Fisher.

Fonte: Elaboração dos autores.

Alguns estudos associam a anemia e a diminuição das concentrações séricas de hemoglobina ao pior prognóstico de pacientes hospitalizados. Uma pesquisa envolvendo 422 cães em estado crítico constatou que um volume globular abaixo de $29,5 \%$, concentração de hemoglobina menor que $11 \mathrm{~g} / \mathrm{dL}$ e leucócitos totais abaixo de $10.300 / \mathrm{mm}^{3}$ estavam associados a menor sobrevivência (RABELO; ARNOLD; ALSUA, 2009). Tais associações não foram observadas nesse trabalho, talvez pela amostragem inferior avaliada (Tabela 6).

Tabela 6. Associação do desfecho com as variáveis hematológicas, em média e mediana (Q3-Q1), para cães hospitalizados, no período de agosto de 2011 a agosto de 2012, no setor de Clínica Médica de Animais de Companhia, do Hospital Veterinário da Universidade Estadual de Londrina. Londrina, 2013.

\begin{tabular}{cccc}
\hline Variável & $\begin{array}{c}\text { G1 (Alta) } \\
\mathrm{n}=126\end{array}$ & $\begin{array}{c}\text { G2 (Óbito) } \\
\mathrm{n}=59\end{array}$ & Análise \\
\hline Volume globular ${ }^{1}$ & & & \\
Média $\pm \mathrm{DP}$ & $31,32 \pm 13,56$ & $27,4 \pm 10,12$ & $\mathrm{M}-\mathrm{W} *^{*}$ \\
Mediana & $32,1(42,2-20,0)$ & $24,7(33,6-19,4)$ & $\mathrm{p}=0,071$ \\
Hemoglobina & & & \\
Média $\pm \mathrm{DP}$ & $9,57 \pm 4,0$ & $8,5 \pm 3,4$ & $\mathrm{M}-\mathrm{W}$ \\
Mediana & $10,0(13,0-6,5)$ & $8,3(10,5-5,9)$ & $\mathrm{p}=0,071$ \\
Linfócitos $^{3}$ & & & \\
Média $\pm \mathrm{DP}$ & $2230,2 \pm 4404,4$ & $1469,4 \pm 1408,6$ & $\mathrm{M}-\mathrm{W}$ \\
Mediana & $1412,4(2607,0-742,0)$ & $1058,0(1882,0-485,0)$ & $\mathbf{p}=\mathbf{0 , 0 2 7}$ \\
\hline
\end{tabular}

${ }^{1}$ Valores de Referência: 37 a 55\% (BUSH, 2004).

${ }^{2}$ Valores de Referência: 12 a 18 g/dL (BUSH, 2004).

${ }^{3}$ Valores de Referência: 1.000 a $4.800 / \mu \mathrm{L}$ (BUSH, 2004).

*Teste de Mann-Whitney.

Fonte: Elaboração dos autores.

O número de linfócitos indica a capacidade de defesa imunológica do organismo (CHANDRA, 1992). Entretanto, seus valores diminuem em decorrência de vários fatores, como tratamento com esteroides, imunossupressores, infecções, estresse metabólico, entre outros. (JEEJEEBHOY,
2000; ACOSTA; GOMEZ-TELLO; SANTANA, 2005; OLIVEIRA; ROCHA; SILVA, 2008). Os animais estudados, G1 e G2, apresentaram número total de linfócitos dentro dos valores de referência, entretanto os animais do G2 apresentaram valores significativamente menores (Tabela 6). A menor 
quantidade de linfócitos nos animais que foram a óbito sugere menor competência imunológica e, portanto, maior susceptibilidade a agentes infecciosos (CHANDRA, 1992; BUSH, 2004). Outra justificativa para valores de G2 seria a possibilidade de ocorrência de maior estresse metabólico nesse grupo (ACOSTA; GOMEZTELLO; SANTANA, 2005; OLIVEIRA; ROCHA; SILVA, 2008).

O fibrinogênio é uma proteína de fase aguda sintetizada pelo fígado, cuja concentração plasmática eleva-se sob a ação de várias citocinas e quimocinas envolvidas no processo inflamatório (ANDREWS;
REAGAN; DeNICOLA, 1994). Há trabalhos que afirmam que a determinação do fibrinogênio é tão significativa para cães, como marcador de inflamação aguda, como é para equinos (SUTTON; JOHNSTONE, 1977; ANDREWS; REAGAN; DeNICOLA, 1994). Foi hipotetizado que cães com hiperfibrinegemia sofreriam de inflamação mais intensa e teriam pior prognóstico, entretanto, a associação do fibrinogênio com o desfecho dos animais não foi observada neste trabalho, sendo então, considerada má indicadora de prognóstico (Tabela 7). Não foram encontradas outras pesquisas analisando a associação dessa variável, como marcadora de prognóstico de cães.

Tabela 7. Associação do desfecho com a concentração sérica de fibrinogênio, em frequência (\%), para cães hospitalizados, no período de agosto de 2011 a agosto de 2012, no setor de Clínica Médica de Animais de Companhia, do Hospital Veterinário da Universidade Estadual de Londrina. Londrina, 2013.

\begin{tabular}{cccc}
\hline Fibrinogênio $^{1}$ & $\begin{array}{c}\text { G1 (Alta) } \\
\mathrm{n}=113\end{array}$ & $\begin{array}{c}\text { G2 (Óbito) } \\
\mathrm{n}=48\end{array}$ & \multirow{2}{*}{ Análise } \\
\hline $100-500 \mathrm{mg} / \mathrm{mL}$ & $99(87,61 \%)$ & $38(79,17 \%)$ & $\chi^{2}=1,89 *$ \\
$>500 \mathrm{mg} / \mathrm{mL}$ & $14(12,39 \%)$ & $10(20,83 \%)$ & $\mathrm{GL}=1 ; \mathrm{p}=0,168$ \\
\hline
\end{tabular}

${ }^{1}$ Valores de Referência: 100 a 500 mg/L (BUSH, 2004).

$* \chi^{2}=$ Teste de Qui-quadrado, GL = Grau de Liberdade.

Fonte: Elaboração dos autores.

A hiperglicemia é comum após o trauma físico ou em doenças críticas devido à secreção de hormônios, como catecolaminas, cortisol, hormônio do crescimento e glucagon, na resposta ao estresse (MEGUID; COLLIER; HOWARD, 1981; PEREIRA, 2007). Esses hormônios são chamados de diabetogênicos, porque aumentam a resistência dos receptores periféricos à ação da insulina (PEREIRA, 2007; MAICÁ; SCHWEIGERT, 2008). Em humanos, a hiperglicemia está associada com mau prognóstico, aumento do risco de infecções, infarto do miocárdio, polineuropatia e falência múltipla de órgãos (CHASE et al., 2008; PRINS, 2010).
No presente trabalho, os animais G2 evidenciaram valores de glicemia inferiores ao G1 $(p=0,026)$, entretanto, dentro dos valores de referência. Os animais G1 apresentaram média discretamente elevada e mediana normal (Tabela 8). Apesar de a diferença ser estatisticamente significativa $(p=0,026)$, na prática, a glicose não foi um bom indicador de prognóstico, pois os valores encontrados nos grupos pouco divergiram da normalidade. É importante ressaltar que a glicemia pode se alterar devido à doença de base, utilização de alguns fármacos ou por administração intravenosa de glicose, variáveis que não foram controladas nesse estudo (BUSH, 2004). 
Tabela 8. Associação do desfecho com as variáveis bioquímicas, em média e mediana (Q3-Q1), para cães hospitalizados, no período de agosto de 2011 a agosto de 2012, no setor de Clínica Médica de Animais de Companhia, do Hospital Veterinário da Universidade Estadual de Londrina. Londrina, 2013.

\begin{tabular}{|c|c|c|c|}
\hline Variável & $\begin{array}{c}\text { G1 (Alta) } \\
n=126\end{array}$ & $\begin{array}{c}\text { G2 (Óbito) } \\
\mathrm{n}=59\end{array}$ & Análise \\
\hline Glicemia $^{1}$ & & & \multirow{3}{*}{$\begin{array}{c}M-W^{*} \\
\mathbf{p}=\mathbf{0 , 0 2 6}\end{array}$} \\
\hline Média \pm DP & $118,2 \pm 53,8$ & $107,5 \pm 69,9$ & \\
\hline $\begin{array}{l}\text { Mediana } \\
\text { Cálcio }^{2}\end{array}$ & $106,0(124,0-92,0)$ & $97,0(116,0-81,0)$ & \\
\hline Média \pm DP & $11,9 \pm 1,1$ & $11,7 \pm 0,9$ & \multirow{2}{*}{$\begin{array}{c}M-W \\
\mathbf{p}=\mathbf{0 , 0 1 3}\end{array}$} \\
\hline $\begin{array}{l}\text { Mediana } \\
\text { Fósforo }^{3}\end{array}$ & $12,1(12,6-11,7)$ & $11,8(12,4-11,4)$ & \\
\hline Média \pm DP & $6,27 \pm 4,23$ & $7,63 \pm 4,56$ & \multirow[t]{2}{*}{$\begin{array}{c}M-W \\
\mathbf{p}=\mathbf{0 , 0 3 0}\end{array}$} \\
\hline $\begin{array}{c}\text { Mediana } \\
\text { Colesterol }^{4}\end{array}$ & $5,42(6,82-4,02)$ & $6,52(9,06-4,40)$ & \\
\hline Média \pm DP & $209,6 \pm 74,9$ & $221,3 \pm 98,9$ & \multirow[t]{2}{*}{$\begin{array}{c}M-W \\
p=0,573\end{array}$} \\
\hline $\begin{array}{c}\text { Mediana } \\
\text { Magnésio }^{5}\end{array}$ & $208,5(267,0-152,0)$ & $206,0(296,0-147,0)$ & \\
\hline Média \pm DP & $2,7 \pm 0,3$ & $2,6 \pm 0,3$ & \multirow[t]{2}{*}{$\begin{array}{c}M-W \\
p=0,672\end{array}$} \\
\hline $\begin{array}{c}\text { Mediana } \\
\text { Albumina }\end{array}$ & $2,7(3,0-2,5)$ & $2,7(3,0-2,4)$ & \\
\hline Média \pm DP & $3,0 \pm 0,5$ & $2,6 \pm 0,6$ & \multirow[t]{2}{*}{$\begin{array}{c}\mathrm{M}-\mathrm{W} \\
\mathbf{p}=\mathbf{0 , 0 0 4}\end{array}$} \\
\hline $\begin{array}{c}\text { Mediana } \\
\text { Proteína Total }^{7}\end{array}$ & $3,0(3,3-2,7)$ & $2,8(3,2-2,1)$ & \\
\hline Média \pm DP & $6,9 \pm 1,5$ & $6,4 \pm 1,8$ & \multirow[t]{2}{*}{$\begin{array}{r}M-W \\
\mathbf{p}=\mathbf{0 , 0 2}\end{array}$} \\
\hline Mediana & $7,0(7,9-6,0)$ & $6,3(7,6-5,1)$ & \\
\hline
\end{tabular}

${ }^{1}$ Valores de Referência: 60 a $110 \mathrm{mg} / \mathrm{dL}$ (BUSH, 2004).

${ }^{2}$ Valores de Referência: 8,0 a 12,0 mg/dL (BUSH, 2004).

${ }^{3}$ Valores de Referência: 2,6 a 6,2 mg/dL (BUSH, 2004).

${ }^{4}$ Valores de Referência: 100 a 300 mg/dL (BUSH, 2004).

${ }^{5}$ Valores de Referência: 1,8 a 2,4 mg/dL (BUSH, 2004).

${ }^{6}$ Valores de Referência: 2,6 a 3,3 g/dL (BUSH, 2004).

${ }^{7}$ Valores de Referência: 6,0 a 8,0 g/dL (BUSH, 2004).

*Teste de Mann-Whitney.

Fonte: Elaboração dos autores.

O cálcio possui elevado potencial para predizer o prognóstico (FIGUEIREDO, 2011). Nesse trabalho, foi mensurado o cálcio total. Os animais G2 possuíam médias e medianas de calcemia inferiores aos animais G1, o que foi estaticamente significativo $(p=0,013)$ (Tabela 8). Outras pesquisas analisaram o potencial do cálcio ionizado como indicador de prognóstico em cães traumatizados e constataram menor sobrevivência dos animais hipocalcêmicos (FIGUEIREDO, 2011; HOLOWAYCHUK; MONTEITH, 2011). Os autores ainda relataram que o cálcio ionizado se mostrou relacionado aos valores de cálcio total e foi sugerida a associação entre nível de hipocalcemia com a gravidade da doença 
de base (HOLOWAYCHUK; MONTEITH, 2011). Outro estudo, que avaliou a concentração do cálcio ionizado em cães com doenças críticas, evidenciou a associação entre hipocalcemia com maior tempo de internação, mas não com maior ocorrência de óbito (HOLOWAYCHUK et al., 2009).

O fósforo sérico costuma estar baixo em pacientes críticos em virtude das interações com fármacos e da elevada síntese de ATP, para sustentar a condição de hipermetabolismo (LABADARIOS, 1990; PRINS, 2010). Contrariamente, no presente estudo, notou-se que os animais de $\mathrm{G} 2$ evidenciaram hiperfosfatemia enquanto os animais de G1 apresentaram valores normais (Tabela 8). Isso ocorreu devido à alta prevalência de insuficiência renal (IR) na população estudada. A IR gera hiperfosfatemia, decorrente da menor taxa de filtração glomerular e está frequentemente associada a pior prognóstico (SEGEV; KASS; FRANCEY, 2008).

Segundo alguns autores, o colesterol pode ser empregado para avaliação do prognóstico, níveis séricos baixos estão correlacionados com o aumento da mortalidade e da permanência hospitalar (ACOSTA; GOMEZ-TELLO; SANTANA, 2005; SAMPAIO; FIGUEIREDO, 2005). Apesar disso, neste trabalho, as concentrações dessa variável foram semelhantes e estavam dentro do intervalo de referência nos dois grupos, não sendo evidenciada a associação com o desfecho dos pacientes (Tabela 8).

Em relação ao proteinograma, a diminuição da concentração da proteína total e da albumina é sugestiva de lesão, inflamação e estresse metabólico (DALEY; BISTRIAN, 1994; HARDIE et al., 1995; JEEJEEBHOY, 2000; OLIVEIRA; ROCHA; SILVA, 2008). Em especial, a albumina é considerada marcadora sensível de morbidade, mortalidade e tempo de hospitalização, sendo que, quanto mais grave é a doença, menor é a concentração de albumina e pior é o prognóstico (DALEY; BISTRIAN, 1994; HARDIE et al.,
1995; JEEJEEBHOY, 2000; HIGGINS et al., 2006; OLIVEIRA; ROCHA; SILVA, 2008).

Neste trabalho, foi constatado que os animais que foram a óbito apresentaram concentrações de albumina e proteína total próximas do limite inferior de referência e os animais que tiveram alta exibiram concentrações estatisticamente superiores $(\mathrm{p}=0,027)$ (Tabela 8). Esses resultados corroboram com a literatura vigente (HIGGINS et al., 2006).

Em síntese, os autores não consideraram a glicemia, cálcio, colesterol e proteína total como indicadores de prognóstico, por ter havido pouca variação entre os grupos alta e óbito e em relação aos valores de referência.

Sobre às variáveis eletroforéticas, houve associação do desfecho apenas com as frações alfa2 e betaglobulinas. É importante notar que ambos os grupos (G1 e G2) apresentaram diminuição da fração albumina, porém, com valores inferiores nos animais G2, reforçando a associação entre hipoalbuminemia e mortalidade (Tabela 9).

Em relação à fração alfa 2-globulinas, houve aumento significativo no $\mathrm{G} 1$ em relação ao G2, com concentrações discretamente elevadas (Tabela 9). A banda alfa-2 é constituída por um grupo variado de proteínas, entre elas: haptoglobina, alfa-2-macroglobulina, pré-betalipoproteínas, ceruloplasmina, eritropoetina e a colinesterase (LARSON, 1974; MCPHERSON, 1999; ECKERSALL, 2008; TORRES FILHO, 2008). A alfa-2-macroglobulina e a haptoglobina correspondem à maior parte dessa banda (LARSON, 1974; MCPHERSON, 1999). Essas são, em maioria, proteínas de fase aguda, que modulam o processo inflamatório. $\mathrm{O}$ aumento dessa fração sugere infecção, inflamação ou distúrbios imunológicos, especialmente nos seguintes casos: síndrome nefrótica, diabetes mellitus, doenças agudas, pneumonia, estresse, uso de corticoides, entre outros (LARSON, 1974; MCPHERSON, 1999; THOMAS, 2000; TORRES FILHO, 2008). 
Tabela 9. Associação do desfecho com as variáveis eletroforéticas, em média e mediana (Q3-Q1), para cães hospitalizados, no período de agosto de 2011 a agosto de 2012, no setor de Clínica Médica de Animais de Companhia, do Hospital Veterinário da Universidade Estadual de Londrina. Londrina, 2013.

\begin{tabular}{|c|c|c|c|}
\hline Variável & $\begin{array}{c}\text { G1 (Alta) } \\
n=126\end{array}$ & $\begin{array}{c}\text { G2 (Óbito) } \\
n=55\end{array}$ & Análise \\
\hline Albumina ${ }^{1}$ & & & \multirow{3}{*}{$\begin{array}{l}M-W * \\
p=0,074\end{array}$} \\
\hline Média \pm DP & $2,21 \pm 0,77$ & $1,98 \pm 0,84$ & \\
\hline $\begin{array}{c}\text { Mediana } \\
\text { Alfa-1 globulina }\end{array}$ & $2,14(2,71-1,63)$ & $1,80(2,64-1,41)$ & \\
\hline Média \pm DP & $0,33 \pm 0,43$ & $0,28 \pm 0,42$ & \multirow[t]{2}{*}{$\begin{array}{c}\mathrm{M}-\mathrm{W} \\
\mathrm{p}=0,251\end{array}$} \\
\hline $\begin{array}{c}\text { Mediana } \\
\text { Alfa-2 globulina }^{3}\end{array}$ & $0,21(0,35-0,10)$ & $0,18(0,32-0,09)$ & \\
\hline Média \pm DP & $1,28 \pm 0,80$ & $0,94 \pm 0,74$ & \multirow[t]{2}{*}{$\begin{array}{c}M-W \\
\mathbf{p}=\mathbf{0 , 0 0 5}\end{array}$} \\
\hline $\begin{array}{c}\text { Mediana } \\
\text { Betaglobulina }^{4}\end{array}$ & $1,23(1,93-0,60)$ & $0,92(1,34-0,19)$ & \\
\hline Média \pm DP & $1,41 \pm 0,70$ & $1,10 \pm 0,68$ & \multirow[t]{2}{*}{$\begin{array}{c}M-W \\
\mathbf{p}=\mathbf{0 , 0 0 3}\end{array}$} \\
\hline $\begin{array}{c}\text { Mediana } \\
\text { Gamaglobulina }\end{array}$ & $1,36(1,81-0,96)$ & $0,99(1,45-0,60)$ & \\
\hline Média \pm DP & $1,72 \pm 1,60$ & $2,14 \pm 1,79$ & \multirow[t]{2}{*}{$\begin{array}{c}M-W \\
p=0,101\end{array}$} \\
\hline Mediana & $1,16(2,36-0,63)$ & $1,69(3,11-0,79)$ & \\
\hline
\end{tabular}

${ }^{1}$ Valores de Referência: 2,5 a 4,0 g/dL (BUSH, 2004).

${ }^{2}$ Valores de Referência: 0,2 a 0,5 g/dL (BUSH, 2004).

${ }^{3}$ Valores de Referência: 0,3 a 1,1 g/dL (BUSH, 2004).

${ }^{4}$ Valores de Referência: 1,2 a 2,2 g/dL (BUSH, 2004).

${ }^{5}$ Valores de Referência: 0,8 a 1,8 g/dL (BUSH, 2004).

*Teste de Mann-Whitney.

Fonte: Elaboração dos autores.

A princípio, a elevação da fração alfa 2-globulina no grupo que obteve alta parece controverso, mas, os autores acreditam que tal fato sugere que houve resposta orgânica adequada frente à doença de base. Animais em estado crítico, como no G2, poderiam estar sofrendo de desnutrição proteica severa a ponto de não sintetizarem essas proteínas em quantidade adequada (BRUNETTO, 2007). Outra possibilidade seria o maior acometimento dos animais de G1 por doenças agudas, o que explicaria o aumento da fração alfa2-globulinas. Não foram encontrados outros trabalhos associando os dados de eletroforese ao prognóstico de cães.

Em relação à fração betaglobulina, o grupo G2 apresentou valores abaixo da referência enquanto o
G1 apresentou concentrações normais (Tabela 9). As betaglobulinas são um grupo heterogêneo de proteínas, cujo as principais são: beta-lipoproteínas, hemopexina, transferrina, componente C3 do complemento, ferritina, proteína C-reativa, IgA, IgM, beta-2-lipoproteína, plasminogênio e fibrinogênio. Portanto, também envolvem proteínas de fase aguda (LARSON, 1974; MCPHERSON, 1999; ECKERSALL, 2008; TORRES FILHO, 2008). A diminuição dessas proteínas nos animais que foram a óbito sugere ineficiência da reação de fase aguda, desnutrição proteica grave ou perda das proteínas endógenas devido a doença de base, como glomerulopatias ou enteropatias perdedora de proteínas (BRUNETTO, 2006; TORRES FILHO, 2008; PRINS, 2010). 
Alguns fatores como a fluidoterapia, medicações utilizadas e doenças de base dos pacientes podem ter interferido nos resultados laboratoriais destas avaliações. Além disso, as avaliações clínicas como ECC e EMM possuem certo grau de subjetividade. Também não foi possível separar as análises por doenças específicas, sendo que a amostra estudada foi, dessa forma, heterogênea, mas representativa das populações em internamentos veterinários.

\section{Conclusões}

São indicativos de mau prognóstico em cães hospitalizados: baixos valores de ECC (especialmente nas graduações 1 e 2), baixos valores de EMM, número total de linfócitos reduzido, hiperfosfatemia, concentrações reduzidas de albumina e de proteína total e diminuição da fração betaglobunina, na eletroforese. São indicadores de bom prognóstico: ECC elevados (especialmente nas graduações 6 e 7), adequado EMM e elevação da fração alfa2-globulina, na eletroforese.

O aumento das proteínas de fase aguda (alfa2globulina) foi associado a bom prognóstico, enquanto a diminuição (betaglobulinas) foi associada a maior mortalidade.

Apesar de ter sido encontrado associação significativa com o desfecho dos pacientes, os autores não consideraram a glicemia, cálcio e proteína total como bons indicadores de prognóstico.

Outras avaliações contendo número maior de cães hospitalizados devem ser estudados para reforçar ou discordar dos achados deste estudo.

\section{Agradecimentos}

Agradeço as estagiárias que auxiliaram na coleta de dados: Priscila dos Santos, Talita Bianca Brunharo, Ana Carolina Buzzo, Caroline Spera. Ao Conselho Nacional de Desenvolvimento Científico e Tecnológico (CNPQ) e Hospital Veterinário da
Universidade Estadual de Londrina, por financiarem o projeto.

\section{Referências}

ACOSTA, E. J.; GOMEZ-TELLO, V.; SANTANA, R. S. Nutritional assessment of the severely ill patient. Nutrición Hospitalaria, Madrid, v. 20, n. 2, p. 5-8, 2005.

ALVES, C. J.; FRANCO, P. P.; NAKATA, C. T.; COSTA, G. L. G.; GENARO, M. S.; AGOSTINE, G.; LUZ, J. L.; LEITE, M. F. M. Avaliação de índices prognósticos para pacientes idosos admitidos em unidades de terapia intensiva. RBTI, Revista Brasileira de Terapia Intensiva, São Paulo, v. 21, n. 1, p. 1-8, 2009.

ANDREWS, D. A.; REAGAN, W. J.; DeNICOLA, D. B. Plasma fibrinogen in recognizing equine inflammatorty disease. Continuing Education for the Practicing Veterinarian, Yardley, v. 16, n. 10, p. 1349-1357, 1994.

BATEMAN, S. W.; CHEW, D. J. Fluid therapy for dogs and cats. In:__. Saunders manual of small animal practice. $3^{\text {th }}$ ed. St. Louis: Saunders Elsevier, 2006. cap. 5, p. 85 .

BEGHETTO, M. G. Estado nutricional como preditor de morte, infecção e permanência hospitalar. Porto Alegre: Universidade Federal do Rio Grande do Sul, 2007. 202 p.

BRUNETTO, M. A. Avaliação de suporte nutricional sobre a alta hospitalar em cães e gatos. Jaboticabal: Universidade Estadual Paulista Júlio de Mesquita Filho, 2006. 86 p.

. Imunotrição: o papel da dieta no restbelecimento das defesas naturais. Acta Scientiae Veterinariae, Porto Alegre, v. 35, n. 2, p. s230-s232, 2007.

BUSH, B. M. Nutrientes e Metabólicos. In: Interpretação de resultados laboratoriais para clínicos de pequenos animais. São Paulo: Roca, 2004. cap. 5, p.180-191.

CERVI, A.; FRANCESCHINI, S. C.; PRIORI, S. E. Análise crítica do uso de índice de massa corporal para idosos. Revista de Nutrição, Campinas, v. 6, n. 18, p. 765-75, 1998.

CHANDLER, M. L.; GRECO, D. S.; FETTMAN, M. J. Hypermetabolism in illness and injury. Compendium on Continuing Education for the Practicing Veterinarian, Yardley, v. 14, n. 10, p. 1284-1290, 1992.

CHANDRA, R. K. Protein energy malnutrition and immunological responses. Journal of Nutrition, Bethesda, v. 122, n. 3, p. 597-600, 1992. 
CHASE, J. G.; SHAW, G.; LE COMPTE, A.; LONERGAN, T.; WILLACY, M.; WONG, X. W.; LIN, J.; LOTZ, T.; LEE, D.; HANN, C. Implementation and evaluation of the SPRINT protocol or tight glycaemic control in critically ill patients: a clinical practice change. Critical Care, Brussels, v. 2, n. 12, p. 49-61, 2008.

COCKCROFT, P. D. Clinical reasoning and decision analysis. Veterinary Clinics of Noth America - Small Animal Practice, Amsterdam, v. 37, n. 3, p. 499-520, 2007.

DALEY, B. J.; BISTRIAN, B. R. Nutritional assessment. In: ZALOGA, G. P. (Ed.). Nutritional in critical care. St. Louis: Mosby, 1994. p. 9-33.

ECKERSALL, P. D. Proteins, proteomics and the dysproteinemias. In: KANEKO, J. J.; HARVEY, J. W.; BRUSS M. L. Clinical biochemistry of domestic animals. $6^{\text {th }}$ ed. Burlington: Academic Press, 2008. p. 117-155.

EVANS, W. J.; MORLEY, J. E.; ARGILÉS, J.; BALES, C.; BARACOS, V.; GUTTRIDGE, D.; JATOI, A.; KALANTAR-ZADEH, K.; LOCHS, H.; MANTOVANI, G.; MARKS, D.; MITCH, W. E.; MUSCARITOLI, M.; NAJAND, A.; PONIKOWSKI, P.; FANELLI, F. R.; SCHAMBELAN, M.; SCHOLS, A.; SCHUSTER, M.; THOMAS, D.; WOLFE, R.; ANKER, S. D. Cachexia: a new definition. Clinical Nutrition, Texas, v. 6, n. 27, p. 793-799, 2008.

FERREIRA, M. M.; SCIALOM, J. M.; CAMPOS, A. D.; RAMALHO, L. L. Z.; MARCHINI, J. S.; FÉRES, O.; ROCHA, J. J. R. Efeito da desnutrição na cicatrização de anastomoses colônicas: estudo experimental em Ratos. Revista Brasileira de Coloproctologia, Rio de Janeiro, v. 26, n. 3, p. 239-243, 2006.

FIGUEIREDO, I. H. Hipocalcémia: quantificação do cálcio ionizado inicial em cães traumatizados e seu valor predictivo. Lisboa: Universidade Técnica de Lisboa, 2011. 55 p.

FONTOURA, C. S. M; CRUZ, D. O.; LONDERO, L. G.; VIEIRA, R. M. Avaliação nutricional de paciente critico. Revista Brasileira de Terapia Intensiva, São Paulo, v. 18, n. 3, p. 298-306, jul./set. 2006.

HARDIE, E. M.; JAYAWICKRAMA, J.; DUFF, L.C.; BECKER, K. M. Prognostic indicators of survival in high-risk canine surgery patients. Journal of Veterinary Emergency and Critical Care, London, v. 5, n. 1, p. 4249, 1995.

HIGGINS, P. A.; DALY, B. J.; LIPSON, A. R.; SU-ER, G. Assessing nutritional status in chronically ill adult patients. American Journal of Critical Care, New York, v. 15, n. 1, p. 1-99, 2006.
HOLOWAYCHUK, M. K.; HANSEN, B. D.; DEFRANCESCO, T. C.; MARKS, S. L. Ionized hypocalcemia in critically ill dogs. Journal of Veterinary Internal Medicine, Melbourne, v. 23, n. 3, p. 509-513, 2009.

HOLOWAYCHUK, M. K.; MONTEITH, G. Ionized hypocalcemia as a prognostic indicator in dogs following trauma. Journal of Veterinary Emergency and Critical Care, London, v. 21, n. 5, p. 521-530, 2011.

JEEJEEBHOY, K. N. Nutritional assessment. Nutrition, Syracuse, v. 2, n. 16, p. 585-590, 2000.

KANEKO, J. J.; SMITH, H. The estimation of plasma fibrinogen and its clinical significance in the dog. The California Veterinarian, California, v. 21, n. 4, p. 21-24, 1967.

KING, L. G.; WOHL, J. S.; MANNING, A. M.; HACKNER, S. G.; RAFFE, M. R.; MAISLIN, G. Evaluation of the survival prediction index as a model of risk stratification for clinical research in dogs admitted to intensive care units at four locations. American Journal of Veterinary Research, Schaumburg, v. 62, n. 6, p. 948954, 2001.

LABADARIOS, D. Guidelines for evaluating nutritional status. In: LABADARIOS D.; HAFFEJEE, A. (Ed.). A pocket manual of clinical nutrition. Tygerberg: A SASPEN publication, 1990. v. 1, p. 129-139.

LAFLAMME, D. P. Development and validation of a body condition score system for dogs. Canine Practice, Santa Barbara, v. 22, n. 4, p. 10-15, 1997.

LARSON, P. H. Serum proteins: diagnostic significance of electrophoretic patterns. Human Pathology, Madison, v. 5, n. 6, p. 629-40, nov. 1974.

LOGAN, S.; HILDEBRANDT, L. A. The use of prealbumin to enhance nutrition-intervention screening and monitoring of the malnourished patient. Nutrition Today, Boston, v. 38, n. 5, p. 134-135, 2003.

MAICÁ, A.O.; SCHWEIGERT, I. D. Avaliação nutricional em pacientes graves. Revista Brasileira de Terapia Intensiva, São Paulo, v. 20, n. 3, p. 286-295, 2008.

MCPHERSON, R. A. Proteínas específicas. In: HENRY, J. B. Diagnósticos clínicos e tratamento por métodos laboratoriais. 18. ed. São Paulo: Manole, 1999. p. 24560.

MEGUID, M. M.; COLLIER, M. D.; HOWARD, L. J. Uncomplicated and stressed starvation. Surgical Clinics of North America, Maryland Heights, v. 61, n. 3, p. 52943, jun. 1981. 
MEIJERS, J. M. M.; SCHUEREN, M. A. E. B.; SCHOLS, J. M. G. A.; SOETERS, P. B.; HALFENS, R. J. G. Defining malnutrition: mission or mission impossible? Nutrition, Texas, v. 4, n. 26, p.432-440, 2010.

MICHEL, K. E. Using a diet history to improve adherence to dietary recommendations. Compendium: Continuing Education for Veterinarians, Yardley, v. 18, n. 31, p. 2224, jan. 2009

MINNE, L.; ABU-HANNA, A.; DE JONGE, E. Evaluation of SOFA-based models for predicting mortality in the ICU: a systematic review. Critical Care, Brussel, v. 12, n. 6, p. 161, 2008.

MONDINI, L.; MONTEIRO, C. A. Relevância epidemiológica da desnutrição e da obesidade em distintas classes sociais: métodos de estudo e aplicação à população brasileira. Revista Brasileira de Epidemiologia, São Paulo, v. 1, n. 1, p. 28-39, 1998.

MONTEIRO, P. O. A.; VICTORA, C. G.; BARROS, F. C.; TOMASI, E. Diagnóstico de sobrepeso em adolescentes: estudo do desempenho de diferentes critérios para o índice de massa corporal. Revista de Saúde Pública, São Paulo, v. 34, n. 5, p. 506-513, 2000.

MULLER, J. E.; PINHEIRO, M. S.; MENDONÇA, D. C Adaptação do índice de massa corporal humano para cães. Ciência Rural, Santa Maria, v. 38, n. 4, p. 10381043, jul. 2008.

OLIVEIRA, L. M. L.; ROCHA, A. P. C.; SILVA, J. M. A. Avaliação nutricional em pacientes hospitalizados: uma responsabilidade interdisciplinar. Revista Saber Científico, Porto Velho, v. 1, n. 1, p. 1-10, 2008.

PEREIRA, S. Efeito da desnutrição na resposta imune ao stress. Revista da Associação Portuguesa dos Nutricionistas, Porto, v. 5, n. 7, p. 21-22, 2007.

POSES, R. M.; MCCLISH, D. K.; BEKES, C.; SCOTT, W. E.; MORLEY, J. N. Ego bias, reverse ego bias, and physicians' prognostic. Critical Care Medicine, Mount Prospect, v. 19, n. 12, p. 1533-1539, 1991.

PRINS, A. Nutritional assessment of the critically ill patient. South African Journal of Clinical Nutrition, Lyttelton, v. 23, n. 1, p. 11-18, 2010.

RABELO, R. C.; ARNOLD, C. F. A evolução da medicina prognóstica na medicina veterinária intensiva. Clínica Veterinária, São Paulo, v. 15, n. 78, p. 20-26, 2009.

RABELO, R. C.; ARNOLD, C. F.; ALSUA, S. C. RICO Score - classificação rápida de sobrevida em cuidados intensivos. Variáveis inter-relacionadas em cães. Clínica Veterinária, São Paulo, v. 15, n. 78, p. 28-38, 2009.

REILLY, H. M.; MARTINEAU, J. K.; MORAN, A.; KENNEDY, H. Nutritional screening: evaluation and implementation of a simple Nutrition Risk Score. Clinical Nutrition, Texas, v. 5, n. 14, p. 269-273, 1995.

REMILLARD, R. L.; ARMSTRONG, P. J.; DAVENPORT, D. J. da. Assisted feeding in hospitalized patients: enteral and parenteral nutrition. In: HAND, M. S.; TRATCHER, C. D.; REMILLARD, R. L. Small animal clinical nutrition. $4^{\text {th }}$ ed. Topeka: Mark Morris Institute, 2000. p. 351-390.

SAMPAIO, L. R.; FIGUEIREDO, V. C. Correlação entre o índice de massa corporal e os indicadores antropométricos de distribuição de gordura corporal em adultos e idosos. Revista de Nutrição, Campinas, v. 18, n. 1, p. 53-61, 2005.

SEGEV, G.; KASS, P. H.; FRANCEY, T. A novel clinical scoring system for outcome prediction in dogs with acute kidney injury managed by hemodialysis. Journal of Veterinary Internal Medicine, Melbourne, v. 22, n. 2, p. 301-308, 2008.

SILVA, L. M. S.; MARTINS, L. F.; SANTOS, M. C. F. C.; OLIVEIRA, R. M. Índices prognósticos na prática clínica de enfermagem em terapia intensiva: revisão integrativa. Revista Eletrônica de Enfermagem, Goiânia, v. 1, n. 16, p. 179-190, jan./mar. 2014

SPEROTTO, F. M.; SPINELLI, R. B. Avaliação nutricional em idosos independentes de uma instituição de longa permanência no município de Erechim-RS. Revista Perspectiva, Erechim, v. 34, n. 125, p. 105-116, mar. 2010.

SUTTON, R. H.; JOHNSTONE, M. The value of plasma fibrinogen estimations in dogs. A comparison with total leucocyte and neutrophil counts. The Journal of Small Animal Practice, Gloucestershire, v. 4, n. 18, p. 277-281, 1977.

THOMAS, J. S. Overview of plasma proteins. In: FELDMAN, B. F.; ZINKL J. G.; JAIN, N. C. Schalm's veterinary hematology. $5^{\text {th }}$ ed. New York: Lippincott Williams Wilkins, 2000. p. 891-898.

TORRES FILHO, H. M. Eletroforese de proteínas. Richet Nouvelles, Rio de Janeiro, v. 11, n. 3, p. 1-8, set. 2008.

VANNUCCHI, H.; UNAMUNO, M. R. D. L; MARCHINI, J. S. Avaliação do estado nutricional. Medicina, Ribeirão Preto, v. 29, n. 1, p. 5-18, jan./mar. 1996.

WORLD SMALL ANIMAL VETERINARY ASSOCIATION. Nutrional assesment guidelines. Ontario: WSAVA Global Veterinary Development, 2011. 12 p. Disponível em: <http://www.wsava.org/guidelines/ global-nutrition-guidelines $>$. Acesso em: 11 set. 2014. 